\title{
Accelerator Challenges and Opportunities for Future Neutrino Experiments
}

\author{
Michael S. Zisman \\ Center for Beam Physics, Accelerator \& Fusion Research Division \\ Lawrence Berkeley National Laboratory, Berkeley, CA 94720 U.S.A.
}

\begin{abstract}
There are three types of future neutrino facilities currently under study, one based on decays of stored betaunstable ion beams ("Beta Beams"), one based on decays of stored muon beams ("Neutrino Factory"), and one based on the decays of an intense pion beam ("Superbeam"). In this paper we discuss the challenges each design team must face and the R\&D being carried out to turn those challenges into technical opportunities. A new program, the Muon Accelerator Program, has begun in the U.S. to carry out the R\&D for muon-based facilities, including both the Neutrino Factory and, as its ultimate goal, a Muon Collider. The goals of this program will be briefly described.
\end{abstract}

Keywords: Beta Beams, Neutrino Factory, Superbeam, neutrino beam, ionization cooling PACS: 29.20.D-, 29.27.Eg, 29.25.-t

\section{INTRODUCTION}

The discovery of neutrino oscillations has led to a strong interest in providing intense beams of accelerator-produced neutrinos. Such facilities may be able to observe, for the first time, CP violation in the lepton sector, a phenomenon that could explain the existence of our matter-dominated universe.

Several ideas for providing the required neutrino beams are being explored worldwide:

- a Beta Beam facility based on decays of a stored beam of beta-unstable ions

- a Neutrino Factory based on the decays of a stored muon beam (which could serve as the precursor to an energy-frontier Muon Collider)

- a Superbeam based on the decays of an intense pion beam

Each of these approaches has its advantages and disadvantages. All of them are challenging, and it is likely that all will be expensive. The EUROnu study [1] will attempt to compare all of these options on an equal footing.

\section{PHYSICS CONTEXT}

Beta Beams arise from the decays

$$
\begin{aligned}
& { }^{6} \mathrm{He} \rightarrow{ }^{6} \mathrm{Li}+\mathrm{e}^{-}+\bar{v}_{e} \text { and } \\
& { }^{18} \mathrm{Ne} \rightarrow{ }^{18} \mathrm{~F}+\mathrm{e}^{+}+v_{e} .
\end{aligned}
$$

This baseline scenario produces low-energy neutrinos. Neutrino Factory beams arise from the decays

$$
\begin{aligned}
& \mu^{-} \rightarrow \mathrm{e}^{-} \bar{v}_{e} v_{\mu} \text { and } \\
& \mu^{+} \rightarrow \mathrm{e}^{+} \bar{v}_{\mu} v_{\mathrm{e}} .
\end{aligned}
$$

In each case the neutrinos are high energy and there are equal numbers of electron antineutrinos and muon neutrinos (or vice versa) produced.

For a Neutrino Factory the electron neutrinos are the most favorable, as the $v_{\mathrm{e}} \rightarrow v_{\mu}$ oscillations give rise to easily detectable "wrong sign" muons. A Superbeam based on pion decay does not provide electron neutrinos.

\section{FACILITY DESCRIPTIONS}

A common feature of all future neutrino beam facilities is the requirement for substantially increased intensity compared with existing facilities. This feature manifests itself in two ways - the need for very intense particle sources and the need for very large detectors. Both represent major technical challenges and extend the state-of-the-art by a factor of 5-10. The demand for high intensity is exacerbated by the fact that all neutrino production schemes rely on the decays of secondary, or even tertiary, beams. 


\section{Beta Beam}

Several versions of Beta Beam facilities have been considered, but the present baseline [2] for the EUROnu study (see Fig. 1) is based on beams of ${ }^{6} \mathrm{He}$ and ${ }^{18} \mathrm{Ne}$ with $\gamma=100$, with the beam aimed toward Fréjus. The CERN PS and SPS accelerators are part of the acceleration chain, and the SPL and Linac 4 are used for ion production. A newly constructed decay ring, with $C=6.9 \mathrm{~km}$ is required, as are an ISOL target, pulsed ECR ion source, low-energy ion linac and rapid cycling synchrotron to bring the ions to an energy suitable for PS injection.

\section{Neutrino Factory}

The Neutrino Factory baseline layout from the IDS-NF study [3] is shown in Fig. 2. It comprises a $4 \mathrm{MW}$ proton driver; a free Hg-jet target with a solenoidal capture and decay channel; a bunching and phase rotation section to reduce the energy spread of the beam; a transverse ("4D") cooling channel to reduce the muon beam emittance; an acceleration system consisting of a linac, a pair of recirculating linear accelerator (RLAs), and a non-scaling fixed-field, alternating gradient (FFAG) ring to bring the muons to an energy of $25 \mathrm{GeV}$; and a pair of decay rings with long straight sections aimed at detectors some 3000 and $7500 \mathrm{~km}$ distant from the rings. A detailed description of the facility can be found in [4].

An alternative, low-energy Neutrino Factory design [5] is being explored at Fermilab. Such a facility is well-matched to the Fermilab-to-DUSEL distance, and is expected to be less costly than the baseline design shown in Fig. 2. It may be advantageous if the value of $\sin ^{2} 2 \theta_{13}$ is sufficiently large.

\section{Superbeam}

A Superbeam facility is simply a (much) higher power version of today's conventional neutrino beam facilities. The approach is, in some sense, evolutionary rather than revolutionary, but the increased beam power makes it nonetheless a big step forward. The

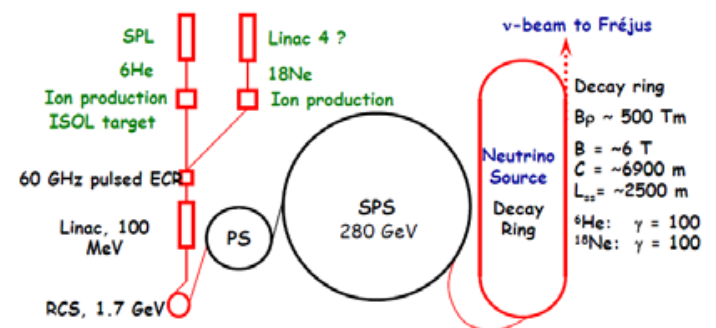

FIGURE 1. Schematic of Beta Beam baseline configuration using CERN facilities,

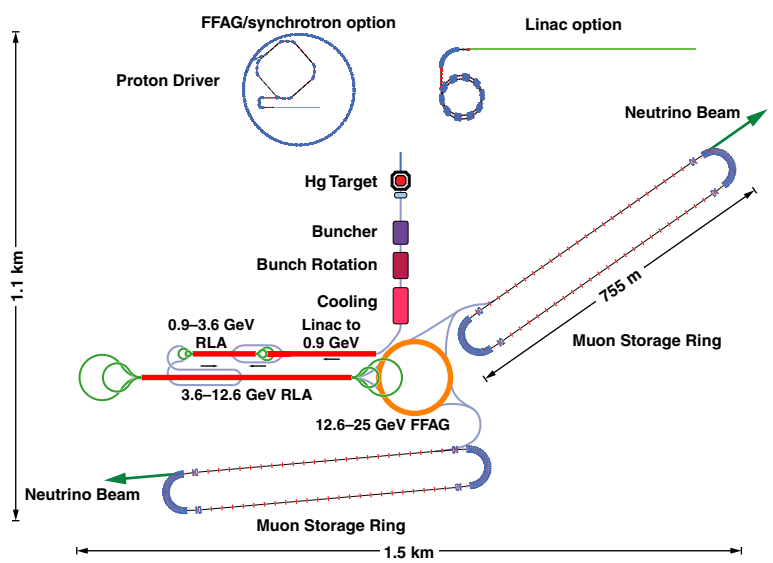

FIGURE 2. Schematic of Neutrino Factory baseline configuration.

version being studied by the EUROnu Work Package 2 team, with a baseline from CERN to Fréjus, is illustrated schematically in Fig. 3

\section{TECHNICAL CHALLENGES}

All three of the facilities described above entail significant technical challenges. Turning these challenges into opportunities is accomplished with intense and dedicated R\&D efforts.

\section{Beta Beam}

For a Beta Beam facility, the most daunting challenge is to deliver the isotopes needed at the required intensity. Delivery involves several steps-initial production, transport to the ion source, ionization, and bunching. Based on present EUROnu studies [6], it appears to be straightforward to provide a ${ }^{6} \mathrm{He}$ beam; ${ }^{18} \mathrm{Ne}$ appears challenging, but a concept based on the ${ }^{19} \mathrm{~F}(\mathrm{p}, 2 \mathrm{n})$ reaction is under study and looks promising. Other challenges include the various RF manipulations required to transport the beams through the chain of accelerators, beam losses that degrade the beam pipe vacuum, and RF stacking in the decay ring. All of these appear to be manageable.

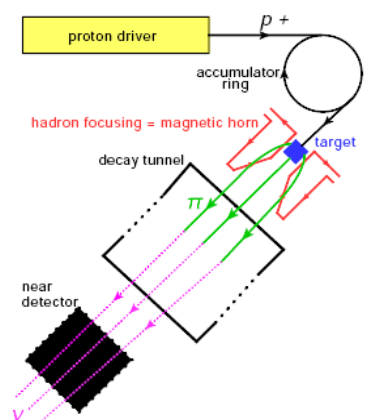

FIGURE 3. Schematic of EUROnu Superbeam configuration. 


\section{Neutrino Factory}

The challenges of a Neutrino Factory are by now wellknown. In brief:

- muons are created as a tertiary beam ( $\rightarrow \pi$ $\rightarrow \mu$ ), which results in (i) a low production rate for usable muons and hence the need for a target that can tolerate multi-MW of protons; and (ii) a beam with a large energy spread and large phase space area and hence the need for emittance cooling and a high-acceptance acceleration system

- muons have a very short lifetime (2.2 $\mu \mathrm{s}$ at rest), which requires rapid beam manipulations and hence the need for ionization cooling [7] using high-gradient RF cavities in a magnetic field, followed by a very rapid acceleration system

One challenge for a Neutrino Factory is the need for very short proton bunches. To get $4 \mathrm{MW}$ of protons at $10 \mathrm{GeV}$ and $50 \mathrm{~Hz}$ implies an intensity of $5 \times 10^{13}$ $\mathrm{p} /$ pulse. To minimize the muon intensity loss, the proton bunches should ideally be only 1 ns rms length, which is difficult to achieve at high intensity and modest energy.

A target that can tolerate such a beam is likewise a challenge. The baseline design for the Neutrino Factory [4] makes use of a Hg-jet target, as illustrated in Fig. 4; work on a "powder jet" target (see Fig. 5) is also ongoing [8].

As is by now well-known, operating normalconducting RF cavities in a strong axial magnetic field has been found to be difficult [9]. This configuration is required for the cooling channel in a Neutrino Factory, and thus presents a challenge. Based on present knowledge, the degradation in gradient in a cooling channel configuration is a serious, but not a "fatal," problem. Throughput decreases by about 20\% when the channel gradient is reduced from $15 \mathrm{MV} / \mathrm{m}$ to $10 \mathrm{MV} / \mathrm{m}$. Past experiments have suffered from breakdown in the cavity coupler region, a problem that will be corrected in the next round of testing.

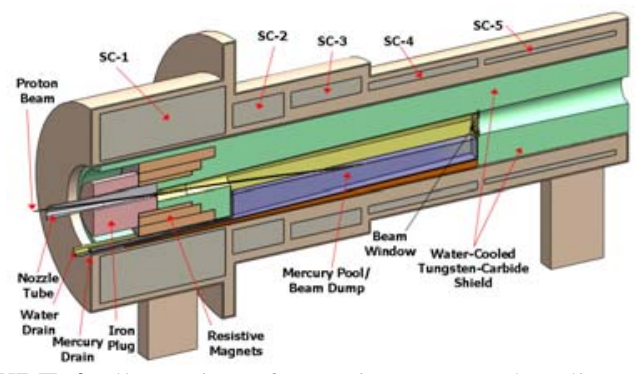

FIGURE 4. Illustration of Neutrino Factory baseline Hg-jet target configuration.

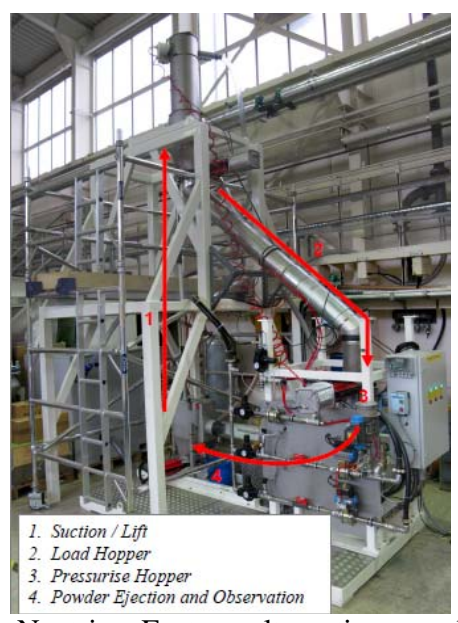

FIGURE 5. Neutrino Factory alternative powder-jet target R\&D setup at RAL.

\section{Superbeam}

The main challenges for a Superbeam facility relate to the high intensity requirement. In particular, one needs:

- a target capable of handling $4 \mathrm{MW}$ of protons

- horns that handle $4 \mathrm{MW}$ of protons at $50 \mathrm{~Hz}$ Another important parameter is beam purity, which demands good charge selection from the horn system.

Because the target is situated in close proximity to the horn, spatial constraints favor a solid, or possibly a powder target. Materials compatibly issues make the use of a mercury target impractical. The target environment is a difficult one. Providing adequate cooling is not easy, and, while the need for repairs in this area is inevitable, the high-radiation environment renders hands-on maintenance impossible.

The approach to dealing with these issues is to use four low- $Z$ solid targets, each with its own horn, as illustrated in Fig. 6. This choice reduces both the power deposition in the target and the horn repetition rate by a factor of four. The present plan calls for single-horn optics (that is, no reflector) with an optimized horn shape.

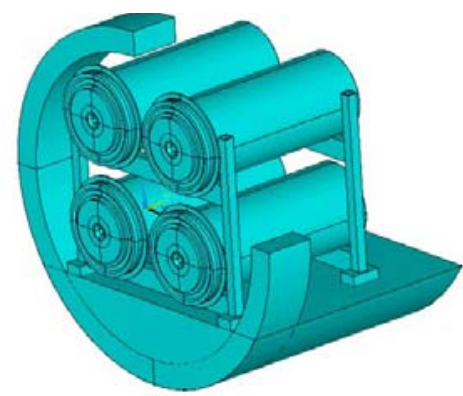

FIGURE 6. Concept of EUROnu Superbeam multiple target and horn configuration. The efficiency of this system is essentially the same as that for a single horn design. 


\section{R\&D ACTIVITIES}

To transform the above challenges into opportunities, worldwide R\&D efforts are under way. For the Beta Beam, the main items are ion production, evaluation of collective effects, and RF issues. For the Neutrino Factory, target design, cooling issues, and RF performance in a magnetic field are the main $R \& D$ focus. For the Superbeam, the main items are the target and the horn design and performance.

\section{Beta Beam}

One new concept being explored is the use of ions having high- $Q$ beta decays, namely ${ }^{8} \mathrm{Li}$ and ${ }^{8} \mathrm{~B}$. Design of an ionization cooling ring is being studied [10] and a lattice has been developed. Present estimates indicate that obtaining the requisite ion intensities would require a gas-jet target thickness some $10^{4}$ times that of existing jet targets, so there is still work to do before adopting the new scheme.

Production cross sections for several key reactions are being measured as part of the EUROnu effort to firm up intensity estimates. Studies of ${ }^{6} \mathrm{Li}\left({ }^{3} \mathrm{He}, \mathrm{n}\right)^{8} \mathrm{~B}$ will be made using the RipeN detector at Legnaro [11], and plans are being developed to measure the ${ }^{19} \mathrm{~F}(\mathrm{p}, 2 \mathrm{n}){ }^{18} \mathrm{Ne}$ cross section using a target with a molten NaF cooling loop [6].

Lastly, ion source technology is being pursued in several places, including the SEISM source in France $[12,13]$ and a $37 \mathrm{GHz}$ Gyrotron source [14] in Russia.

\section{Neutrino Factory}

Neutrino Factory R\&D has three main thrusts:

- $\quad$ simulations and theory

- technology development, including a highpower target, cooling channel components, and rapid acceleration techniques

- system tests of target and cooling systems

Recent simulation effort, carried out in conjunction with the IDS-NF, has focused on simplifying designs to optimize performance and reduce costs. The primary challenge for technology development is the operation of high-gradient RF cavities in a strong axial field [15]. System test work, involving a substantial international collaboration, is currently aimed at demonstrating ionization cooling of muons in the Muon Ionization Cooling Experiment (MICE) [16].

The MICE goals are to:

- design, engineer and build a section of a realistic cooling channel

- measure its performance in a muon beam under a variety of conditions
- compare the results with simulation codes

The experiment, which comprises one cell of the Study 2 [17] cooling channel along with upstream and downstream spectrometer solenoids with scintillating fiber tracking detectors, is situated at RAL in the UK. As shown in Fig. 7, the civil engineering is nearly complete, except for the installation of the RF power infrastructure. All major hardware components for the experiment are in construction, although there have been some delays in reaching the performance specifications for the cryo-cooled magnet systems.

Another R\&D activity is the EMMA experiment at Daresbury Laboratory [18] to test an electron model of a non-scaling FFAG ring. The $C=16.6 \mathrm{~m}$ ring (see Fig. 8) will be used to investigate the longitudinal dynamics, transmission, emittance growth, and influence of resonances in this novel type of accelerator. Commissioning of the ring is under way.

\section{Superbeam}

The apparatus required is basically a known quantity, but some R\&D for the horn and its power supply are planned.

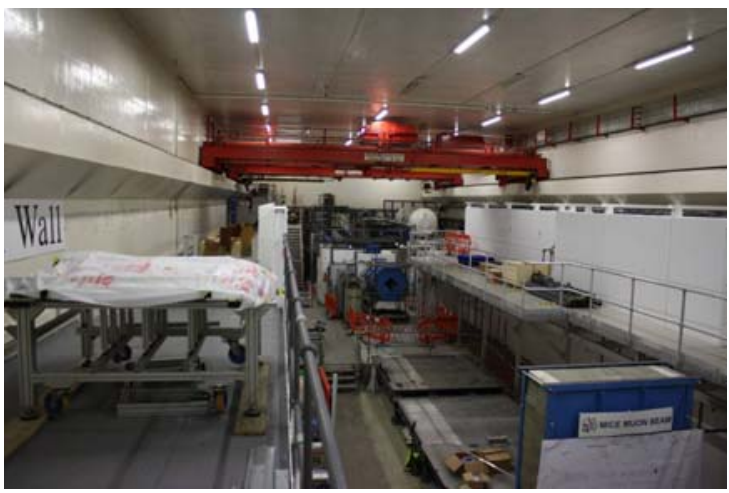

FIGURE 7. View of MICE hall at RAL looking upstream toward the final beam line quadrupole. The muon beam dump appears in the foreground. The cooling channel hardware will be sited in the region in between.

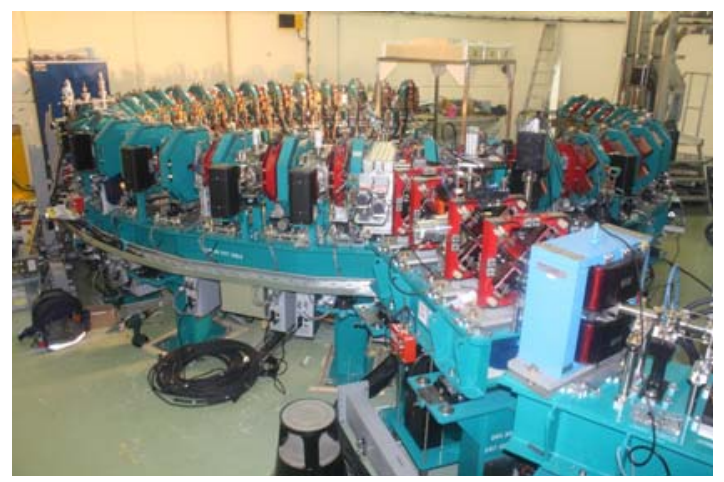

FIGURE 8. View of EMMA ring at Daresbury Laboratory. 


\section{MUON ACCELERATOR PROGRAM}

In the U.S., the Neutrino Factory and Muon Collider Collaboration and Fermilab's Muon Collider Task Force have jointly proposed a 7-year R\&D program to the Department of Energy. A successful review of the proposal took place in August 2010, and the new Muon Accelerator Program (MAP) has now begun. Its main deliverables include:

- a Muon Collider Design Feasibility Study

- a Neutrino Factory Reference Design Report, under the auspices of the IDS-NF

- component development and testing, with demonstration of the key technologies

- system tests of $4 \mathrm{D}$ and eventually $6 \mathrm{D}$ cooling, including completion of MICE and carrying out a 6D cooling channel "bench test"

\section{SUMMARY}

In the recent past there has been substantial progress toward the design of accelerator-based neutrino facilities to study CP violation in the lepton sector. This work, which includes the study of high-power targets, novel beam-cooling techniques, ion source development, and rapid acceleration techniques, is markedly extending the state-of-the-art in accelerator science. The R\&D activities outlined briefly here are worldwide efforts that are (voluntarily) coordinated internationally. This is a strength of our program and serves as an excellent model for carrying out such international efforts.

\section{ACKNOWLEDGMENTS}

I wish to thank my colleagues in MAP, MICE, EUROnu, and IDS-NF for sharing both their expertise and their enthusiasm for the research described here.

This work was supported by the Office of Science, U. S. Dept. of Energy, under Contract No. DE-AC0205CH11231.

\section{REFERENCES}

1. See http://www.euronu.org/.

2. E. Wildner, "Beta Beams for Neutrino Production," in AIP Conf. Proc. 1222, Neutrino Factories, Superbeams and Beta Beams, $11^{\text {th }}$ International Workshop, ed. M. C. Goodman, D. M. Kaplan, and Z. Sullivan, p. 31 (2010).

3. See https://www.ids-nf.org/wiki/FrontPage/Accelerator/ Baselines/1.

4. M. Apollonio et al., "Accelerator Design Concept for Future Neutrino Facilities,” JINST_4_P07001, see http://www.iop.org/EJ/abstract/1748-0221/4/07/P07001.
5. S. Geer, O. Mena, and S. Pascoli, Phys. Rev. D $\underline{75}$, 093001 (2007); A. Bross et al., Phys. Rev. D 구, 093012 (2008).

6. T. Stora, "Baseline Ion Production Dedicated to Beta Beams,” http://heplnv135.pp.rl.ac.uk/joomla/index.php? option=com_docman\&task=doc_download\&gid=46\&Ite mid $=5$.

7. D. Neuffer, Part. Acc. 14, 75 (1983).

8. C. J. Densham et al., "The Potential of Fluidized Powder Technology in High Power Accelerator Facilities," in Proc. 2009 Particle Accelerator Conf., Vancouver, BC, Canada, May 4-8, 2009, p. 1833; see http://accelconf.web.cern.ch/AccelConf/PAC2009/papers /we1grc04.pdf

9. D. Stratakis, J. C. Gallardo, and R. B. Palmer, "RF Breakdown in Magnetic Fields: Previous Work, Recent Theory, and Future Plans,” in AIP Conf. Proc. 1222, Neutrino Factories, Superbeams and Beta Beams, $11^{\text {th }}$ International Workshop, ed. M. C. Goodman, D. M. Kaplan, and Z. Sullivan, p. 303 (2010).

10. E. Benedetto, "Ionization Cooling in a Low-Energy Ion Ring with Internal Target for Beta Beams Production,” in Proc. 2010 International Particle Accelerator Conf., Kyoto, Japan, May 23-28, 2010, p. 849.

11. See http://www.lnl.infn.it/ spesweb/index.php/researchon-nuclear-physics/143-ripen.

12. L. Latrasse et al., "SEISM: A $60 \mathrm{GHz}$ Cusp Electron Cyclotron Resonance Ion Source,” Rev. Sci. Instr. 1ㅗ, 02A324 (2010).

13. T. Thuillier et al., "High Frequency ECR Ion Source (60 $\mathrm{GHz}$ ) in Preglow Mode for Bunching of Beta-Beam Isotopes,” PoS (NuFact08)089.

14. V.G. Zorin et al., "Gasdynamic ECR Sources of Multicharged Ions,” in Proc. $18^{\text {th }}$ Intl. Workshop on ECR Ion Sources, Chicago, IL, Sept. 15-18, 2008, p. 128.

15. D. Huang et al., "RF Studies at Fermilab MuCool test Area," in Proc. 2009 Particle Accelerator Conf., Vancouver, BC, Canada, May 4-8, 2009, p. 888; see http://accelconf.web.cern.ch/AccelConf/PAC2009/papers /tu5pfp032.pdf.

16. L. Coney, "Status of the MICE Muon Ionization Cooling Experiment,” in Proc. 2009 Particle Accelerator Conf., Vancouver, BC, Canada, May 4-8, 2009, p. 1680; see http://accelconf.web.cern.ch/AccelConf/PAC2009/papers /tu6rfp057.pdf.

17. S. Ozaki, R. Palmer, M. Zisman, and J. Gallardo, eds., "Feasibility Study-II of a Muon-Based Neutrino Source," BNL-52623 (2001); see http://www.cap.bnl.gov/mumu/ studyii/final_draft/The-Report.pdf.

18. S. L. Smith, "EMMA - the World's First Non-scaling FFAG Accelerator," in Proc. 2009 Particle Accelerator Conf., Vancouver, BC, Canada, May 4-8, 2009, p. 1947; http://accelconf.web.cern.ch/AccelConf/PAC2009/papers /we4pbi01.pdf. 


\section{DISCLAIMER}

This document was prepared as an account of work sponsored by the United States Government. While this document is believed to contain correct information, neither the United States Government nor any agency thereof, nor the Regents of the University of California, nor any of their employees, makes any warranty, express or implied, or assumes any legal responsibility for the accuracy, completeness, or usefulness of any information, apparatus, product, or process disclosed, or represents that its use would not infringe privately owned rights. Reference herein to any specific commercial product, process, or service by its trade name, trademark, manufacturer, or otherwise, does not necessarily constitute or imply its endorsement, recommendation, or favoring by the United States Government or any agency thereof, or the Regents of the University of California. The views and opinions of authors expressed herein do not necessarily state or reflect those of the United States Government or any agency thereof or the Regents of the University of California. 